\title{
ON SOME ASYMPTOTIC PROPERTIES OF POISSON PROCESS
}

\author{
TAKEYUKI HIDA
}

The Poisson process $X(t, \omega),{ }^{1}{ }^{\prime}(\omega \in \Omega, 0 \leqq t<\infty)$, as is well-known, is a temporally and spatially homogeneous Markoff process satisfying

$$
\begin{gathered}
X(0, \omega)=0 \text { and } X(t, \omega)=\text { integer } \geqslant 0 \text { for every } \omega \in \Omega, \\
\operatorname{Pr}\left\{X(t, \omega)-X\left(t^{\prime}, \omega\right) \geq k\right\}=\sum_{i=k}^{r} \frac{\left\{\lambda\left(t-t^{\prime}\right)\right\}^{i}}{i !} e^{-\lambda\left(t-t^{\prime}\right)} \text { for } t>t^{\prime},
\end{gathered}
$$

where $k$ is a non-negative integer and $\lambda$ is a positive constant. In this note we consider the random variable $L_{m}(\omega)$ which denotes the length of $t$-interval such that $X(t, \omega)=m(m=0,1,2, \ldots)$ and some of other properties concerning them.

$\S 1$. The known results on $\mathbf{L}_{m}$.

Definition. We define $L_{m}(\omega)$, the function of $m$ and $\omega$, as follows,

where

$$
L_{m}(\omega)=t_{m+1}(\omega)-t_{m}(\omega),
$$

$$
t_{m}(\omega)=\operatorname{Min}\{\tau ; X(\tau, \omega)=m\} .
$$

This $t_{m}(\omega)$ exists almost certainly by the right continuity property of Poisson process, and furthermore it is clear that $t_{m}(\omega)$ is measurable. Thus $L_{m}(\omega)$ becomes a non-negative random variable.

THEOREM 1. $L_{0}, L_{1}, \ldots, L_{m}, \ldots$ are mutually independent random variables with a common distribution function $F(l)$, where

\section{Furthermore}

$$
F(l)= \begin{cases}1-e^{-\lambda l} & \text { if } l \geq 0 \\ 0 & \text { otherwise. }\end{cases}
$$

This theorem was already suggested by P. Levy $[2]^{3)}$ and a rigorous proof was

Received March 19, 1953.

1) $\omega$ denotes the probability parameter.

2) $E(\ldots)$ and $V(\ldots)$ denote the mean and the variance respectively.

3) Numbers in brackets refer to the bibliography at the end of this note. 
given by T. Nishida [1]. From this theorem we can easily conclude the following corollaries.

COROLLARY 1. The characteristic function $\varphi_{L}(z)$ of $L_{m}$, and therefore that of $F$, is $\frac{\lambda e^{i z}}{\lambda-i z}$.

COROLlaRY 2. The probability $L_{m} \geqslant l\left(l_{0}\right)$ under the assumption $L_{m} \geqslant l_{0}$ is $e^{-\lambda\left(l-l_{0}\right)}$ and its conditional expectation is $\frac{1}{\lambda}+l_{0}$.

$\S 2$. The definitions and the behaviours of $M_{n}$ and $m_{n}$

Definition. Let $M_{n}$ be defined by

$$
M_{n}(\omega)=\max \left\{L_{0}(\omega), L_{1}(\omega), \ldots, L_{n-1}(\omega)\right\} .
$$

$M_{n}(\omega)$ is monotone non-decreasing with respect to $n$ for every $\omega$. The probability law of $M_{n}(\omega)$ is easily obtained as follows:

$$
\begin{aligned}
& \operatorname{Pr}\left\{M_{n}<x\right\} \quad\left(=\operatorname{Pr}\left\{M_{n} \leqq x\right\}\right) \\
& =\operatorname{Pr}\left\{L_{0}<x, L_{1}<x, \ldots, L_{n-1}<x\right\} \\
& =\operatorname{Pr}\left\{L_{0}<x\right\} \operatorname{Pr}\left\{L_{1}<x\right\} \ldots \operatorname{Pr}\left\{L_{n-1}<x\right\} \\
& \text { (as } L_{m} \text { is mutually independent) } \\
& =\left(1-e^{-\lambda x}\right)^{n} .
\end{aligned}
$$

THEOREM 2. $E\left(M_{n}\right)=O(\log n)$.

Proof. We have

$$
\begin{aligned}
E\left(M_{n}\right) & =n \lambda \int_{0}^{\infty} x e^{-\lambda x}\left(1-e^{-\lambda x}\right)^{n-1} d x \\
& =-\frac{1}{\lambda} n \int_{0}^{\infty} \log \left(1-e^{-y}\right) e^{-n y} d y \\
& =-\frac{1}{\lambda} n \int_{0}^{\varepsilon}-\frac{1}{\lambda} n \int_{\varepsilon}^{\infty}
\end{aligned}
$$

where $\varepsilon$ is arbitrary small such that $1-e^{-y} \sim y$ when $0 \leqq y \leqq \varepsilon$. The second term is $o(\log n)$ when $n \rightarrow \infty$, and

$$
\begin{aligned}
-n \int_{0}^{\varepsilon} \log y e^{-n y} d y & =\log n \int_{0}^{n \varepsilon}\left(1-\frac{\log z}{\log n}\right) e^{-z} d z \\
& =O(\log n) .
\end{aligned}
$$

Hence we can conclude $E\left(M_{n}\right)=O(\log n)$.

THEOREM 3. $\lambda M_{n} ! \log n$ converges in law to the random variable $Y$ which takes the value 1 with probability 1.

Proof. We have

$$
\operatorname{Pr}\left\{\lambda M_{n} / \log n<x\right\}=\left(1-1 / n^{\mathfrak{r}}\right)^{n} \rightarrow\left\{\begin{array}{lll}
1 & \text { if } & x>1 \\
0 & \text { if } & 1>x \geqslant 0
\end{array}\right.
$$


as $n$ tends to $\infty$.

More precisely we may prove

THEOREM 4. If $0<\alpha<1$, then

$$
\operatorname{Pr}\left\{\lim _{n \rightarrow \infty} \inf _{n} \lambda M_{n} / \alpha \log n \geq 1\right\}=1 .
$$

In order to prove the theorem above we need the following lemma.

LEMMA. The series

$$
\sum_{n=1}^{\infty}\left(1-1 / n^{\alpha}\right)^{n}
$$

is convergent when $0<\alpha<1$.

Proof of the Lemma. It is sufficient to prove $u_{n} v_{n} \rightarrow 0 \quad(n \rightarrow \infty)$, where

Let

$$
u_{n}=\left(1-1 / n^{\alpha}\right)^{n}, \quad v_{n}=1 / n^{2} \text {. }
$$

Then

$$
f(x) \equiv x^{2}\left(1-1 / x^{\alpha}\right)^{x}
$$

$$
\begin{aligned}
\log f(x)= & 2 \log x+x \log \left(1-1 / x^{\alpha}\right) \\
= & \frac{(2 \log x) / x+\log \left(1-1 / x^{\alpha}\right)}{1 / x} \\
& \quad \rightarrow \frac{2\left((1-\log x) / x^{2}\right)+\alpha x^{\alpha-1} /\left(1-x^{-\alpha}\right)}{-1 / x^{2}} \quad(x \rightarrow \infty) \\
= & 2(\log x-1)-\alpha x^{-\alpha+1}-1 \\
= & \left(\frac{2(\log x-1)}{x /\left(x^{\alpha}-1\right)}-\alpha\right) \cdot \frac{x}{x^{\alpha}-1} .
\end{aligned}
$$

Here

$$
2 \frac{\log x-1}{x /\left(x^{\alpha}-1\right)} \rightarrow 0, \frac{x}{x^{\alpha}-1} \rightarrow \infty . \quad(x \rightarrow \infty)
$$

Hence $\log f(x) \rightarrow-\infty$ and therefore $f(x) \rightarrow 0$ when $x \rightarrow \infty$. Thus $u_{n} / v_{n} \rightarrow 0$ when $n \rightarrow \infty$.

Proof of Theorem 4. We have, by (6) and by the Lemma above,

$$
\sum_{n=1}^{\infty} \operatorname{Pr}\left\{M_{n}<\frac{\alpha}{\lambda} \log n\right\}=\sum_{n=1}^{\infty}\left(1-\frac{1}{n^{\alpha}}\right)^{n}<\infty .
$$

Therefore, by the Borel-Cantelli's Lemma,

$$
\operatorname{Pr}\left\{\lim _{n \rightarrow \infty} \inf E_{n}^{c}\right\}=1,
$$

where

$$
E_{n}=\left\{\omega ; M_{n}(\omega)<\frac{\alpha}{\lambda} \log n\right\}
$$

On the other hand 


$$
\liminf _{n \rightarrow \infty} E_{n}^{c} \subseteq\left\{\omega ; \liminf _{n \rightarrow \infty} M_{n}(\omega) / \frac{\alpha}{\lambda} \log n \geqq 1\right\} .
$$

This shows that ( 8$)$ is valid.

Definition. Let $m_{n}(\omega)$ be defined by

$$
m_{n}(\omega)=\operatorname{Min}\left\{L_{0}(\omega), L_{1}(\omega), \ldots, L_{n-1}(\omega)\right\} .
$$

$m_{n}(\omega)$ is monotone non-increasing with respect to $n$ for every $\omega$. The law of $m_{n}$ is calculated in the same way as $M_{n}$ :

and hence

$$
\begin{aligned}
& \operatorname{Pr}\left\{m_{n}>x\right\} \quad\left(=\operatorname{Pr}\left\{m_{n} \geqslant x\right\}\right) \\
& \quad=\operatorname{Pr}\left\{L_{0}>x, L_{1}>x, \ldots, L_{n-1}>x\right\} \\
& \quad=\left(e^{-\lambda x}\right)^{n}=e^{-\lambda n x},
\end{aligned}
$$

$$
\operatorname{Pr}\left\{m_{n}<x\right\}=1-e^{-\lambda n x} .
$$

THEOREM 5. If $\beta>1$, then

$$
\operatorname{Pr}\left\{\lim _{n \rightarrow \infty} \sup \lambda m_{n} / \hat{j} n^{-1} \log n \geqslant 1\right\}=0 .
$$

Proof. We have

$$
\operatorname{Pr}\left\{m_{n} \geqslant \beta \log n / \lambda n\right\}=1 / n^{3} \text { and } \sum_{n=1}^{\infty} n^{-3}<\infty .
$$

Thus, by the Borel-Cantelli's Lemma,

where

$$
\operatorname{Pr}\left\{\lim _{n \rightarrow \infty} \sup _{n} F_{n}\right\}=0,
$$

On the other hand

$$
F_{n}=\left\{\omega ; m_{n}(\omega) \geqslant \beta \log n / \lambda n\right\}
$$

$$
\limsup _{n \rightarrow \infty} F_{n} \supseteqq\left\{\omega ; \limsup _{n \rightarrow \infty} \lambda m_{n}(\omega) / \beta n^{-1} \log n \geqq 1\right\} .
$$

Thus we obtain (11).

\section{§ 3. Asymptoric properties of $\mathbf{Z}_{\boldsymbol{n}}$}

Let $Z_{n}(\omega)$ be defined by

$$
Z_{n}(\omega)=\left(L_{0}(\omega)+L_{1}(\omega)+\ldots+L_{n-1}(\omega)\right) / M_{n}(\omega) .
$$

Remembering

$$
\operatorname{Pr}\left\{L_{0}=M_{n}\right\}=\operatorname{Pr}\left\{L_{1}=M_{n}\right\}=\ldots=\operatorname{Pr}\left\{L_{n-1}=M_{n}\right\}=1 / n,{ }^{1)}
$$

we see that $Z_{n}$ has the first and the second (absolute) moments:

$$
\begin{aligned}
E\left(Z_{n}\right)=\int_{0}^{\infty} d x_{1} \cdot n\left(\int_{0}^{\leftarrow n-1} \ldots\right. & \int_{0}^{x_{1}} x_{1}^{x_{1}}+x_{2}+\ldots+x_{n} \\
x_{1} & \times \\
& \left.\times \lambda^{n} e^{-\lambda x_{1}} e^{-\lambda x_{2}} \ldots e^{-\lambda x_{n}} d x_{2} \ldots d x_{n}\right) .
\end{aligned}
$$

1) See e.g. D. A. Darling [3]. 


$$
\begin{aligned}
& E\left(Z_{n}^{2}\right)=\int_{0}^{\infty} d x_{1} \cdot n\left(\int_{0}^{x_{1}} \ldots \int_{0}^{x_{1}}\left(\frac{x_{1}+x_{2}+\ldots+x_{n}}{x_{1}}\right)^{2} \times\right. \\
&\left.\times \lambda^{n} e^{-\lambda x_{1}} e^{-\lambda x_{2}} \ldots e^{-\lambda x_{n}} d x_{2} \ldots d x_{n}\right) .
\end{aligned}
$$

The characteristic function $\varphi_{z_{10}}(z)$ of $Z_{n}$ satisfies

$$
\begin{aligned}
& \varphi_{Z_{13}}(z)=E\left(e^{i z}\left(L_{0}+L_{1}+\ldots+L_{n-1}\right) / M_{n}\right) \\
& =n \lambda^{n} \int_{0}^{\infty} d x_{1}\left(\int_{0}^{x_{1}} \cdots \int_{0}^{x_{1}-1 \text { ple }} e^{i z \frac{x_{1}+x_{2}+\ldots+x_{n}}{x_{1}}} \times\right. \\
& \left.\times e^{-\lambda\left(x_{1}+x_{2}+\ldots+x_{n}\right)} d x_{2} \ldots d x_{n}\right) \\
& =n \lambda^{n} \int_{0}^{\infty} e^{i z-\lambda x_{1}} d x_{1}\left(\int_{0}^{x_{1}} e^{i z \frac{x}{x_{1}}-\lambda x} d x\right)^{n-1}
\end{aligned}
$$

(as $L_{1}, L_{2}, \ldots, L_{n-1}$ are mutually independent)

$$
\begin{gathered}
=n \lambda^{n} \int_{0}^{\infty} e^{i z-\lambda x_{1}} x_{1}^{n-1}\left(\frac{e^{i z-\lambda x_{1}}-1}{i z-\lambda x_{1}}\right)^{n-1} d x_{1} \\
=n \lambda^{n} e^{i z} \int_{0}^{\infty} e^{-\lambda x} x^{n-1}\left(\frac{e^{i z-\lambda x}-1}{i z-\lambda x}\right)^{n-1} d x . \\
\frac{1}{i} \frac{d \varphi_{Z_{n}}(z)}{d z}=n \lambda^{n} e^{i z} \int_{0}^{\infty} e^{-\lambda x} x^{n-1}\left(\frac{e^{i z-\lambda x}-1}{i z-\lambda x}\right)^{n-1} d x \\
+n(n-1) \lambda^{n} e^{i z} \int_{0}^{\infty} e^{-\lambda x} x^{n-1}\left(\frac{e^{i z-\lambda x}-1}{i z-\lambda x}\right)^{n-2} \times \\
\times \frac{(i z-\lambda x) e^{i z-\lambda x}-e^{i z-\lambda x}+1}{(i z-\lambda x)^{2}} d x .
\end{gathered}
$$

$$
\begin{aligned}
\left(\frac{1}{i}\right)^{2} \frac{d^{2} \varphi_{z_{n}}(z)}{d z^{2}}= & n \lambda^{n} e^{i z} \int_{0}^{\infty} e^{-\lambda x} x^{n-1}\left(\frac{e^{i z-\lambda x}-1}{i z-\lambda x}\right)^{n-1} d x \\
+ & 2 n(n-1) \lambda^{n} e^{i z} \int_{0}^{\infty} e^{-\lambda x} x^{n-1}\left(\frac{e^{i z-\lambda x}-1}{i z-\lambda x}\right)^{n-2} \\
& \times \frac{(i z-\lambda x) e^{i z-\lambda x}-e^{i z-\lambda x}+1}{(i z-\lambda x)^{2}} d x \\
+ & n(n-1)(n-2) \lambda^{n} e^{i z} \int_{0}^{\infty} e^{-\lambda x} x^{n-1}\left(\frac{e^{i z-\lambda x}-1}{i z-\lambda x}\right)^{n-3} \\
& \times \frac{\left\{(i z-\lambda x) e^{i z-\lambda x}-e^{i z-\lambda x}+1\right\}^{2}}{(i z-\lambda x)^{4}} d x \\
+ & n(n-1) \lambda^{n} e^{i z} \int_{0}^{\infty} e^{-\lambda x} x^{n-1}\left(\frac{e^{i z-\lambda x}-1}{i z-i x}\right)^{n-2} \\
& \times \frac{(i z-\lambda x)^{2} e^{i z-\lambda x}-2\left\{(i z-\lambda x) e^{i z-\lambda x}-e^{i z-\lambda x}+1\right\}}{(i z-\lambda x)^{3}} d x .
\end{aligned}
$$

The differentiations in (15) and (16) are possible since $Z_{n}$ has the first and the second moments.

Theorem 6. $Z_{n}$ has the first and second absolute moments. And if $n$ is 
sufficiently large, the mean and the standard deviation of $Z_{n}$ are both of order $n ! \log n$.

Proof. The first half of the theorem is proved above. Thus

$$
\begin{aligned}
E\left(Z_{n}\right)= & \frac{1}{i}\left(\frac{d \varphi_{z_{n}}(z)}{d z}\right)_{z=0}=n \lambda^{n} \int_{0}^{\infty} e^{-\lambda x} x^{n-1}\left(\frac{e^{-\lambda x}-1}{-\lambda x}\right)^{n-1} d x \\
+ & n(n-1) \lambda^{n} \int_{0}^{\infty} e^{-\lambda x} x^{n-1}\left(\frac{1-e^{-\lambda x}}{\lambda x}\right)^{n-2} \frac{-\lambda x e^{-\lambda x}-e^{-\lambda x}+1}{\lambda^{2} x^{2}} d x \\
= & \varphi_{Z_{n}}(0)-n(n-1) \lambda \int_{0}^{\infty} e^{-2 \lambda x}\left(1-e^{-\lambda x}\right)^{n-2} d x \\
& +n(n-1) \int_{0}^{\infty} x^{-1} e^{-\lambda x}\left(1-e^{-\lambda x}\right)^{n-1} d x .
\end{aligned}
$$

Here

$$
\begin{aligned}
& \varphi_{Z_{n}}(0)=1 \\
& n(n-1) \lambda \int_{0}^{\infty} e^{-2 \lambda x}\left(1-e^{-\lambda x}\right)^{n-2} d x \\
= & n(n-1) \lambda \int_{0}^{\infty} e^{-\lambda x}\left(1-e^{-\lambda x}\right)^{n-2} d x-n(n-1) \lambda \int_{0}^{\infty} e^{-\lambda x}\left(1-e^{-\lambda x}\right)^{n-1} d x \\
= & n(n-1) \lambda\left(\frac{1}{\lambda(n-1)}-\frac{1}{\lambda n}\right)=1 .
\end{aligned}
$$

The last term of (19), $I_{n}=\int_{0}^{\infty} n(n-1) x^{-1} e^{-\lambda x}\left(1-e^{-\lambda x}\right)^{n-1} d x$, is of order $n / \log n$.

It is proved as follows. We have

$$
I_{n}=\int_{0}^{\infty} \frac{n(n-1) e^{-\lambda . x}}{x}\left(1-e^{-\lambda x}\right)^{n-1} d x=n(n-1) \int_{0}^{\infty} \frac{e^{-n y}}{-\log \left(1-e^{-y}\right)} d y .
$$

Let $a$ be sufficiently small such that $e^{-t} \sim 1-t$ when $0<t<a$, and let $K$ be sufficiently large such that $\log \left(1-e^{-t}\right) \sim e^{-t}$ when $t>K$. Then

$$
\begin{aligned}
& \frac{I_{n}}{n(n-1)}=\int_{0}^{a}+\int_{a}^{K}+\int_{K}^{\infty} \\
\int_{0}^{a} \frac{e^{-n t}}{-\log \left(1-e^{-t}\right)} d t & \sim \int_{0}^{a} \frac{e^{-n t}}{-\log t} d t \\
& =\frac{1}{n \log n} \int_{0}^{n a} \frac{e^{-y}}{1-\log y / \log n} d y \quad(n t=y) \\
& <\frac{1}{n \log n} \int_{0}^{\infty} \frac{e^{-y}}{1-\log y / \log n} d y=O\left(\frac{1}{n \log n}\right),
\end{aligned}
$$

since

$$
\lim _{n \rightarrow \infty} \int_{0}^{\infty} \frac{e^{-y}}{1-\log y / \log n} d y=\int_{0}^{\infty} \lim _{n \rightarrow \infty} \frac{e^{-y}}{1-\log y / \log n} d y=\int_{0}^{\infty} e^{-y} d y=1 .
$$

On the other hand, we have

$$
\frac{1}{n \log n} \int_{0}^{n a} \frac{e^{-y}}{1-\log y / \log n} d y \text { 르 } \frac{1}{n \log n} \int_{1}^{n a} \frac{e^{-y}}{1-\log y / \log n} d y
$$


Hence

$$
\geqslant \frac{1}{n \log n} \int_{1}^{n a} e^{-y} d y=\frac{1}{n \log n}\left(e^{-1}-e^{-n a}\right)
$$

$$
\begin{aligned}
& \int_{0}^{a} \frac{e^{-n t}}{-\log \left(1-e^{-t}\right)} d t=O(1 / n \log n), \\
& \int_{a}^{K} \frac{e^{-n t}}{-\log \left(1-e^{-t}\right)} d t=C\left(e^{-n a}-e^{-n K}\right)=o\left(e^{-n}\right),
\end{aligned}
$$

where $0<1,-\log \left(1-e^{-a}\right) \leqq C \leqq 1 /-\log \left(1-e^{-k}\right)<\infty$.

Therefore

$$
\int_{K}^{\infty} \frac{e^{-n t}}{-\log \left(1-e^{-t}\right)} d t \sim \int_{K}^{\infty} e^{-(n-1) t} d t=o\left(e^{-n}\right)
$$

and thus

This proves

$$
I_{n}=O(n / \log n)
$$

$$
E\left(Z_{n}\right)=O\left(n_{/}^{\prime} \log n\right)
$$

Similarly we have

$$
\begin{aligned}
E\left(Z_{n}^{2}\right) & =\left(\frac{1}{i}\right)^{2}\left(\frac{d^{2} \varphi_{Z_{n}}(z)}{d z^{2}}\right)_{z=0} \\
& =\varphi_{Z_{i i}}(0)+2 n(n-1) \lambda^{2} \int_{0}^{\infty} e^{-\lambda x} x^{n-1}\left(\frac{1-e^{-\lambda x}}{\lambda x}\right)^{n-2} \frac{1-e^{-\lambda x}-\lambda x e^{-\lambda x}}{\lambda^{2} x^{2}} d x \\
& +n(n-1)(n-2) \lambda^{2} \int_{0}^{\infty} e^{-\lambda x} x^{n-1}\left(\frac{1-e^{-\lambda x}}{\lambda x}\right)^{n-3} \frac{\left(-\lambda x e^{-\lambda x}-e^{-\lambda x}+1\right)^{2}}{\lambda^{4} x^{+}} d x \\
& =O(n / \log n)+\frac{n(n-1)(n-2)}{\lambda} \int_{0}^{\infty} \frac{e^{-\lambda x}}{x^{2}}-\left(1-e^{-\lambda x}\right)^{n-3}\left(1-e^{-\lambda x}-\lambda x e^{-\lambda x}\right)^{2} d x \\
& -\frac{n(n-1)}{\lambda} \int_{0}^{\infty} x^{-2} e^{-\lambda x}\left(1-e^{-\lambda x}\right)^{n-2}\left\{\lambda^{2} x^{2} e^{-\lambda x}-2\left(1-e^{-\lambda x}-\lambda x e^{-\lambda x}\right)\right\} d x \\
& =O(n ! \log n)+n(n-1)(n-2) \lambda \int_{0}^{\infty} e^{-3 \lambda x}\left(1-e^{-\lambda x}\right)^{n-3} d x \\
& -2 n(n-1)(n-2) \int_{0}^{\infty} x^{-1} e^{-2 \lambda x}\left(1-e^{-\lambda x}\right)^{n-2} d x \\
& +\frac{n(n-1)(n-2)}{\lambda} \int_{0}^{\infty} x^{-2} e^{-\lambda x}\left(1-e^{-\lambda x}\right)^{n-1} d x \\
& -n(n-1) \lambda \int_{0}^{\infty} e^{-2 \lambda x}\left(1-e^{-\lambda x}\right)^{n-2} d x-2 n(n-1) \int_{0}^{\infty} x^{-1} e^{-2 \lambda x}\left(1-e^{-\lambda x}\right)^{n-2} d x \\
& +\frac{2 n(n-1)}{\lambda} \int_{0}^{\infty} x^{-2} e^{-\lambda x}\left(1-e^{-\lambda x}\right)^{n-1} d x
\end{aligned}
$$

Since

$$
\begin{aligned}
& n(n-1)(n-2) \lambda \int_{0}^{\infty} e^{-3 i x}\left(1-e^{-\lambda x}\right)^{n-2} d x=2, \\
& n(n-1) \lambda \int_{0}^{\infty} e^{-2 \lambda x}\left(1-e^{-\lambda x}\right)^{n-2} d x=1
\end{aligned}
$$

we obtain 


$$
\begin{aligned}
E\left(Z_{n}^{2}\right) & =O(n / \log n)+2 n(n-1)^{2} \frac{1}{\lambda} \int_{0}^{\infty} x^{-2} e^{-\lambda x}\left(1-e^{-\lambda x}\right)^{n-1} d x \\
& -2 n(n-1)^{2} \int_{0}^{\infty} x^{-1} e^{-2 \lambda x}\left(1-e^{-\lambda x}\right)^{n-2} d x
\end{aligned}
$$

Thus we have

$$
\begin{aligned}
V\left(Z_{n}\right) & =E\left(Z_{n}^{2}\right)-\left(E\left(Z_{n}\right)\right)^{2} \\
& =O(n / \log n)+\frac{2 n(n-1)^{2}}{\lambda} \int_{0}^{\infty} x^{-2} e^{-\lambda x}\left(1-e^{-\lambda x}\right)^{n-1} d x \\
& -2 n(n-1)^{2} \int_{0}^{\infty} x^{-1} e^{-2 \lambda x}\left(1-e^{-\lambda x}\right)^{n-2} d x \\
& -\left\{n(n-1) \int_{0}^{\infty} x^{-1} e^{-\lambda x}\left(1-e^{-\lambda x}\right)^{n-1} d x+o(n / \log n)\right\}^{2} \\
& \geq O(n / \log n)+\frac{n(n-1)^{2}}{\lambda} \int_{0}^{\infty} x^{-2} e^{-\lambda x}\left(1-e^{-\lambda x}\right)^{n-1} d x \\
& -2 n(n-1)^{2} \int_{0}^{\infty} x^{-1} e^{-2 \lambda x}\left(1-e^{-\lambda x}\right)^{n-2} d x,
\end{aligned}
$$

since, by the Schwarz's inequality,

$$
\begin{aligned}
\left\{\int_{0}^{\infty} x^{-1} e^{-\lambda x}\left(1-e^{-\lambda x}\right)^{n-1} d x\right\}^{2} & \leqq \int_{0}^{\infty} x^{-2} e^{-\lambda x}\left(1-e^{-\lambda x}\right)^{n-1} d x \int_{0}^{\infty} e^{-\lambda x}\left(1-e^{-\lambda x}\right)^{n-1} d x \\
& =\frac{1}{n \lambda} \int_{0}^{\infty} x^{-2} e^{-\lambda x}\left(1-e^{-\lambda x}\right)^{n-1} d x .
\end{aligned}
$$

Similarly as in the proof of (18), we obtain

$$
\frac{n(n-1)^{2}}{\lambda} \int_{0}^{\infty} \frac{e^{-\lambda x}}{x^{2}}\left(1-e^{-\lambda x}\right)^{n-1} d x=O\left(n^{2} /(\log n)^{2}\right)
$$

There exists a large number $M$ such that

$$
\frac{e^{-2 \lambda x}}{x}\left(1-e^{-\lambda x}\right)^{n-2}<\frac{e^{-\lambda x}}{x^{2}}\left(1-e^{-\lambda x}\right)^{n-1}
$$

whenever $x>M$. This fact implies that $J_{n}=2 n(n-1)^{2} \int_{0}^{\infty} x^{-1} e^{-2 \lambda x}\left(1-e^{-\lambda x}\right)^{n-2} d x$ is, when $n \rightarrow \infty$, negligible in the formula (19). Therefore $E\left(Z_{n}^{2}\right)$ and $V\left(Z_{n}\right)$ are of order $n^{2} /(\log n)^{2}$.

\section{BIBLIOGRAPHY}

[1] T. Nishida, On the inverse function of Poisson process. Mathematica Japonicae. Vol. II No. 3. (1952) pp. 135-142.

[2] P. Lévy, Processus stochastiques et mouvement brownien. (1948) p. 182.

[3] D. A. Darling, The influence of the maximum term in the addition of independent random variables. Transactions of the American Mathematical Society. Vol. 73. No. 1 (1952) pp. 95-107.

[4] T. Öta, On some properties of Poisson process I. Bulletin of the Aichi Gakugei University. Vol. 2. (1953). pp. 1-6.

Mathematical Institute,

Aichi Gakugei University 